\title{
The Effect of Using Educational Computer Games on Recall and Retention of Spelling in Iranian EFL Learners
}

\author{
Hossein Shokri \\ English Department, Science and Research Branch, Islamic Azad University, Gorgan, Iran \\ Seyed Jalal Abdolmanafi-Rokni (Corresponding Author) \\ English Department, Golestan University, Gorgan, Iran \\ E-mail: j.abdolmanafi@gmail.com
}

Received: 07-06-2014

Accepted: 23-07-2014

Published: 01-11-2014

doi:10.7575/aiac.ijalel.v.3n.6p.169

URL: http://dx.doi.org/10.7575/aiac.ijalel.v.3n.6p.169

\begin{abstract}
Applying new methods and techniques for language teaching throughout the history could not address the needs and wants of the learners. Allegedly the reason of this problem laid in contextual factors and learner's considerations including the instrument, medium and also appropriate techniques for instruction. To address this problem, video games were applied for one of the basic steps of language learning and literacy skill for specific age group of 14-16 students i.e. spelling. In this study 40 students from two classes were randomly divided into two groups, control and experimental (each 20). The pre-test was done in one session. After that, the experimental group received the treatment subjecting to original computer games, but the control group received a placebo. The classroom was equipped with the computer, television, overhead projector, etc. Each week was spent on one game. At the end of each session, the students were required to play the game and elicit words and spelling of them. After eight sessions of treatment, through different kinds of video computer games, the results of the study showed that the experimental group outperformed the control group in posttest and delayed posttest. This finding subscribes to the role and efficacy of video computer games on recall and retention of spelling among students. The information provided in this study can be useful for teachers, and material developers.
\end{abstract}

Keywords: video computer games, spelling, recall, retention

\section{Introduction}

In a world divided by clashes of cultures and beliefs, the potential of the new media for fostering intercultural understanding and exchange is enormous. In the history of mankind, access to information and knowledge has never been so straightforward at a local, regional, national, or global level. Judicious deployment of ICTs can encourage and sustain cultural and linguistic diversity in individuals and in a society in general. Facilitating access to other cultures and languages is the chief goal of the language teaching profession, and using ICT resources effectively in their teaching represents one of the chief challenges facing language teachers today.

In language classrooms many students have problems in spelling performance. Possibly spelling, as one of the most important sub skills in basic levels, is confined to a set of limited and controlled question and answer procedure. Applying this view will neglect the key role of spelling as a building block of teaching literacy. We should take this point into account that teaching spelling is a broad area in which there are various opinions involving different procedures and teaching methods.

Some researchers maintain that students can have a good performance at spelling words within sentences rather than in isolation. While others believe that by generalizing visual concepts, or teaching syllabication after spelling learning, students would have a better performance at spelling. Beside these, there are some fundamental strategies that scholars cited, to teach rules inductively through writing, to emphasize using dictionary, to encourage mnemonic devices and spelling tricks for memorization, to predict probable spellings, to teach visualization, and to analyze separately the structural patterns governing the spelling of words (Hinson, 1982).

But the problem is that these studies try to design techniques to improve spelling as a whole. In fact, they did not give any heed to the processes underlying teaching spelling. These processes consisted of retention and recall. The present study attempted to investigate the effectiveness of using computer games on recall and retention of word spelling in Iranian EFL male and female students' spelling.

The present study tries to address one of the basic problems in rudimentary level of language learning. Spelling can be considered as the first techniques in learner's exposure to language classroom. Because of this point, teaching spelling is of great importance. The traditional ways of spelling are not in parallel with modern techniques in language learning. Thus this study assesses the efficacy of computer games over spelling. This study will help teachers and material developers to adopt appropriate techniques for teaching spelling. 
Applying computer games as a technique in class will help teachers to get student's attention completely and make the atmosphere of the class more interactive and attractive. Also, while the students are in basic level, this technique will reduce their stress and help them to get along with the class easily. Beside this, considering computer game playing as a technique in language classroom will help material developers to design techniques which are in parallel with children's and young adult's wants.

There is paucity of researches about using the technologies in improving EFL students' reading, writing, speaking, and listening abilities. The main significance of this research is that this research inspected the effect of technology on EFL students' spelling which is very scarce in the TEFL literature.

\section{Research Questions}

This study strives to answer the following questions:

1. Does computer game have any significant effect on recall of Iranian EFL learners' spelling?

2. Does computer game have any significant effect on retention of Iranian EFL learners' spelling?

\section{Methodology}

\subsection{Participants}

The sample participating in this study consisted of 40 out of a total of 58 male learners in the form of two classes. They were all native speakers of Persian and their age ranged from 14 to 15 . The target population for this study was the two third-grade classes at Sama Middle school in Aliabad Katool, Iran. The participants were divided into two groups of experimental group and control group.

\subsection{Instrumentation}

\subsubsection{Proficiency Test}

The first instrument in this study was Nelson proficiency test. This test was conducted to place students in exact level of proficiency. This test consisted of 45 questions that students should answer the questions in 50 minutes.

\subsubsection{Spelling Test}

The other instruments utilized to collect the data included some games, through which the participants were supposed to improve their spelling. The test consisted of two parts. The first part was Words Unscramble questions and the second part was True or False questions. The test consisted of twenty questions addressing the content of the selected games. The test was used as pretest, posttest and delayed posttest.

\subsection{Validity and Reliability of the Tests}

Three experts checked the validity of the pre-, post, and delayed posttests. The reliability index of the pre-, post, and delayed posttests estimated through the KR-21 formula turned out to be $0.83, .80$ and 0.82 , respectively.

\subsection{Treatment}

The experimental group and control group both received the treatment. The participants in the experimental group were presented with original educational computer games like the control group. During the treatment period the experimental group worked with each game by the teacher in the class. The teacher taught spelling explicitly and consciously to the students. The students had a dictation after each game. The experimental group enjoyed each game a lot. The participants had some home assignments. The participants in the control group were presented with original computer games but the teacher did not teach any spelling. The participants just played the games and they enjoyed the games, but there was not and instruction, dictation and home assignment for them. The contents were practiced and performed via different games like: Hang man, Spelling bee, Fast hand, and Concentration Games.

\subsection{Procedure}

In order to have a homogenous group of the participants in terms of proficiency, the Nelson test was conducted at first. Then the two classes were randomly divided into two groups of experimental group and control group. The research was conducted during the second semester in February, 2014. The pre-test was administered to take up the initial differences among the subjects. The participants were asked to complete pre-test requesting information of spelling knowledge before any game playing began. The treatment was held in the school two sessions a week. The time limit for these sessions was 45 minutes. The participants received 8 sessions of treatment during the semester. The pre-test was done in one session. After pre-test and eight sessions of treatment a post-test was administered to the participants. By comparing pretest and posttest after the treatment, the researcher was able to assess the effects of the computer games on the participants' spelling recall. Next, after one month the participants were required to complete the follow up test (delayed post-test). The pre-test and post-test were administered to assess the participants' recall and the follow up test to assess the participants' retention. Finally, by comparing pre-test and post-tests results with follow up test result, the researcher was able to assess the effects of the computer games on the participants' spelling retention.

\subsection{Data Analysis}

After all tests were administered and the data were collected, to test the research hypotheses and to answer the first and second research questions, t-tests were run on the immediate posttests of spelling recall and spelling retention groups. 


\section{Results}

\subsection{Descriptive Statistics of Variables}

In order to inform the average performance of the participants and distribution of their scores on each of the variables, I attempted to present the descriptive statistics parameters (Mean, Standard deviation, Minimum score, and Maximum score) in Table 1.

Table 1. Mean, Standard Deviation, Minimum Score and Maximum Score

\begin{tabular}{lllllll}
\hline \multirow{3}{*}{ experimental group } & Test & N & Minimum & Maximum & Mean & Std. Deviation \\
& Pretest & 20 & 30 & 36 & 31.45 & 2.012 \\
& posttest & 20 & 37 & 49 & 41.65 & 3.066 \\
\multirow{3}{*}{ control group } & Follow up & 20 & 31 & 45 & 36.60 & 3.515 \\
& Pretest & 20 & 30 & 39 & 31.60 & 2.162 \\
& posttest & 20 & 33 & 44 & 36.05 & 2.373 \\
& Follow up & 20 & 30 & 38 & 32.50 & 2.188 \\
\hline
\end{tabular}

Table 1 presents the experimental and control groups' descriptive statistics in pre-test and post-test. According to Table 1 , the mean score of the experimental group's pre-test is 31.54 that this amount is changed to 41.65 in post-test and 36.60 in the follow up. on the other hand, the mean score of the control group's pre-test is 31.60 that this amount is changed to 36.05 in post-test and 32.50 in the follow up. The data obtained from Table 1, has been illustrated in the Figure 1 in a bar graph.

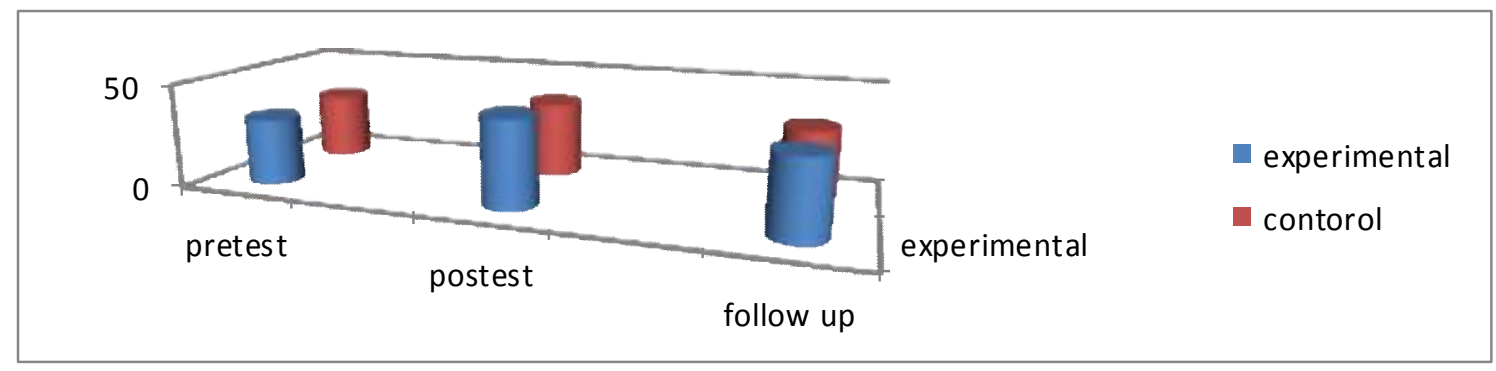

Figure 1. Experimental \& Control Groups’ Mean Scores in Pre-Test, Post-Test, and Follow Up

As it is illustrated in the above bar graph, the students' post-test scores are more than their scores in the pre-test and follow up.

\subsection{Inferential Statistics}

In this part, in order to compare the students in experimental group and control group in pre-test, the independent t-test was utilized.

Table 2. Independent T-Test of Experimental \& Control Groups in Pre-Test

\begin{tabular}{lllllll}
\hline $\mathrm{T}$ & $\mathrm{df}$ & Sig & Mean Difference & $\begin{array}{l}\text { Std. } \\
\text { Difference }\end{array}$ & $\begin{array}{l}\text { Error } \\
\text { 95\% Confidence Interval of the } \\
\text { Difference } \\
\text { Lower }\end{array}$ & $\begin{array}{l}\text { Upper } \\
-0.227\end{array}$ \\
\hline
\end{tabular}

Table 2 presents the results of independent t-test which was used to show the difference between experimental group and control group in the pre-test. The results of the independent t-test showed that there was no significant difference $(p>0.05)$ between the two groups in the pre-test. In other words, the experimental group and control group were equal in level of vocabulary before the treatment.

\subsection{Inspecting Research Questions}

With regard to the first research question, the descriptive statistics showed that there is a difference between control group and experimental group in recall of spelling scores. In order to inspect whether this difference is meaningful or random, the ANOVA analysis was utilized. The results of this analysis have been presented in the Table 3 . 
Table 3. Tests of Between-Subjects Effects

\begin{tabular}{llllll}
\hline Source & Type III Sum of Squares & df & Mean Square & F & Sig. \\
Corrected Model & 375.82 & 2 & 187.91 & 31.14 & .000 \\
Intercept & 63.38 & 1 & 63.38 & 10.50 & 0.003 \\
Pre-test & 62.22 & 1 & 62.22 & 10.31 & 0.003 \\
Group & 323.54 & 1 & 323.54 & 53.61 & 0.000 \\
Error & 223.28 & 37 & 6.03 & \\
Total & 60.972 & 40 & & \\
Corrected Total & 599.10 & 39 & & \\
\hline
\end{tabular}

Based on the results presented in the Table 3, it can be concluded that there is a significant difference between the mean scores of the subjects in two groups in recall of spelling $(\mathrm{F}=53.61, \mathrm{P}<0.01)$. In other words, it can be said that the computer games have a significant influence on EFL students' recall of spelling.

\subsection{Calculation of Effect Sizes (Glass Method)}

The effect size is calculated by dividing the difference between two mean scores (in the pre-test and post-test) of control group and experimental group on the standard deviation of the control group.

The mean of experimental group _ the mean of control group $=5.6$

The standard deviation of the control group $=2.37$

$$
\mathrm{ES}(\text { Effect Size })=\underline{5.6}=2.36
$$

The effect size is 2.36. This effect size shows the experimental intervention (computer games) effect on EFL learners' vocabulary learning which based on Cohen table is considered to be very large.

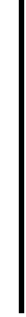

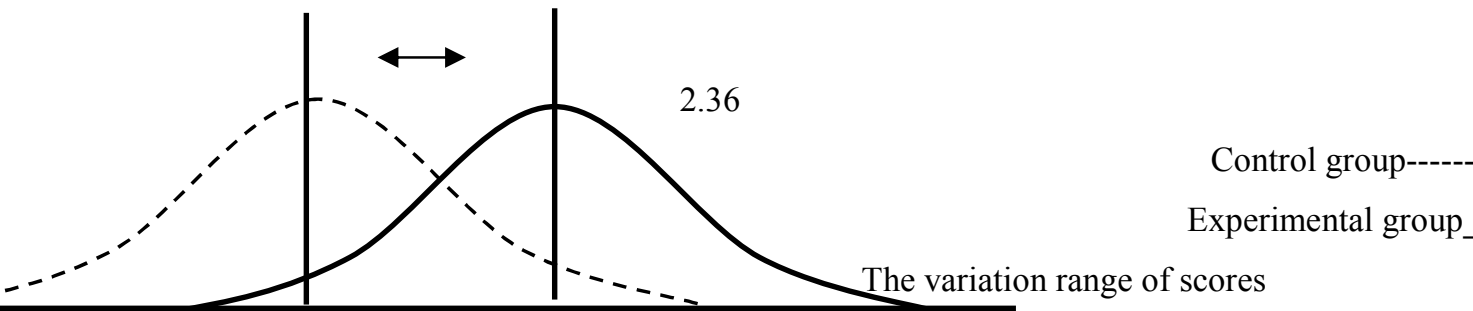

Figure 2. Distribution of Recall of Spelling Scores in Control and Experimental Groups

Figure 2 shows the distribution of the subjects' recall of spelling scores in the experimental and control groups. The difference between the experimental group and the control group is 2.36. This difference based on the standard deviation shows the experimental intervention effects.

With regard to the second research question, the descriptive statistics showed that there is a difference between control group and experimental group in retention of spelling scores. In order to inspect whether this difference is meaningful or random, the ANOVA analysis was utilized. The results of this analysis have been presented in the Table 4.

Table 4. Tests of Between-Subjects Effects

\begin{tabular}{llllll}
\hline Source & Type III Sum of Squares & df & Mean Square & F & Sig. \\
Corrected Model & 174.18 & 2 & 87.09 & 9.80 & .000 \\
Intercept & 86.67 & 1 & 86.67 & 9.75 & 0.003 \\
Pre-test & 22.08 & 1 & 22.08 & 2.48 & 0.12 \\
Group & 156.19 & 1 & 156.19 & 17.57 & 0.000 \\
Error & 328.92 & 37 & 8.89 & & \\
Total & 47700.0 & 40 & & & \\
Corrected Total & 503.10 & 39 & & & \\
\hline
\end{tabular}

Based on the results presented in the Table 4, it can be concluded that there is a significant difference between the mean scores of the subjects in two groups in retention of spelling $(\mathrm{F}=17.57, \mathrm{P}<0.01)$. In other words, it can be said that the computer games have a significant influence on EFL students' retention of spelling.

\subsection{Calculation of Effect Sizes (Glass Method)}

The effect size is calculated by dividing the difference between two mean scores (in the pre-test and post-test) of control group and experimental group on the standard deviation of the control group.

The mean of experimental group _ the mean of control group $=4.1$ 
The standard deviation of the control group $=2.19$

$\mathrm{ES}($ Effect Size $)=\frac{4.1}{2.19}=1.87$

The effect size is 1.87. This effect size shows the experimental intervention (computer games) effect on EFL learners' retention of spelling which based on Cohen table is considered to be very large.

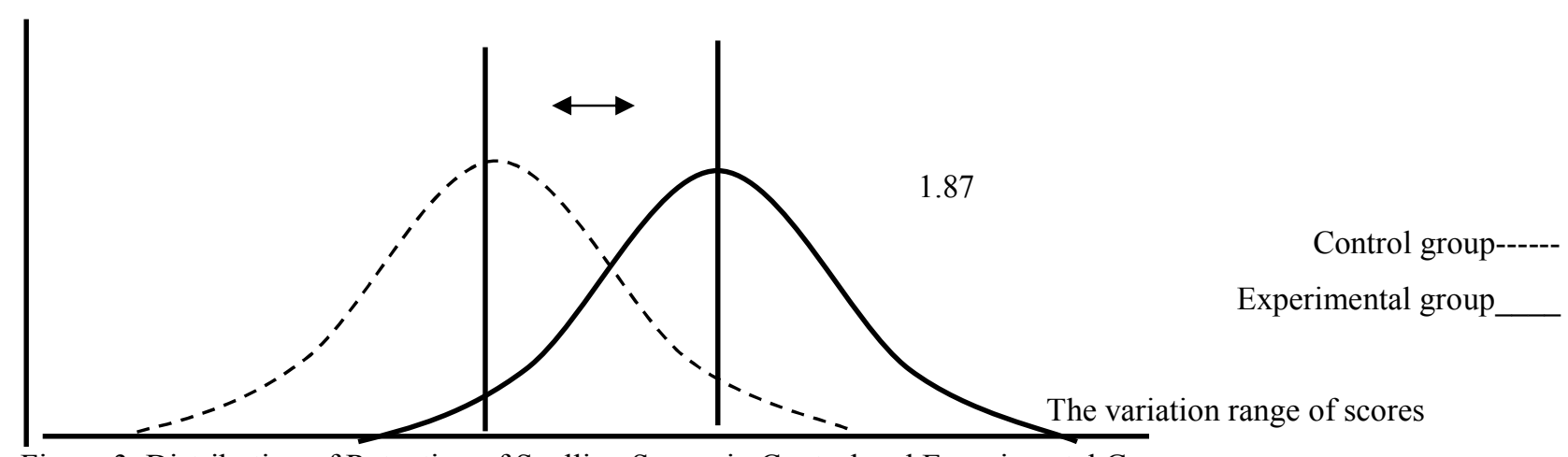

Figure 3. Distribution of Retention of Spelling Scores in Control and Experimental Groups

Figure 3 shows the distribution of the subjects' retention of spelling scores in the experimental and control groups. The difference between the experimental group and the control group is 1.87 of the standard deviation. This difference based on the standard deviation, shows the experimental intervention effects. Based on the presented effect sizes, it can be concluded that the effect of the computer games on students' recall of spelling is more than their retention of spelling.

\section{Findings and Discussion}

The analysis of this empirical study indicates that students predominantly responded favorably to the implementation of computer games for the purpose of enhancing their language learning especially spelling performance. They were naturally curious about applying games for language learning. As Mubireek (2003) reported educators discovered that video games can fit into the educational environment in various ways, ranging from creating total-learning systems to serving as components in a more traditional learning environment. So the atmosphere of this study was very interesting for them. They also highly valued clear links between the computer games and their program of study; learners were more inclined to complete the tasks if they considered them as being relevant to the course's objectives.

Taking into consideration that not all learners are comfortable with technology (Donaldson \& Haggström, 2006), it was also observed that opting for a computer games approach, where regular and direct contact with students was possible, facilitated the development of doing computer game tasks. Training sessions were also organized and feedback confirmed that they who were reluctant to take part in some activities, said that they felt unprepared or were let down by the technology, which highlighted a need to build trust between the users and the tools.

The practicality of these kinds of task through computer games shows that they are like discussion forums, blogs and wikis, "time-and-place-independent communication" technologies (Warschauer, 1997,p. 474), i.e. they are accessible to people around the world at any time, from anywhere (library, computer lab) with an Internet connection or through local networks (Cress \& Kimmerle, 2008). In an educational setting, this extends the learning process outside the scheduled classroom, thus allowing students to catch up with course content even at home.

Psycholinguistically speaking, most of the interactive theories of spelling assume that children use a wide range of spelling strategies from early phases of spelling acquisition on and continue to adaptively use these strategies throughout development (Keuning \& Verhoeven, 2008; Snowling, 1994; Varnhagen, McCallum, \& Burstow, 1997). These are some fundamental strategies that scholars cited, to teach rules inductively through writing, to emphasize using dictionaries, to encourage mnemonic devices and spelling tricks for memorization, to predict probable spellings, to teach visualization, and to analyze separately the structural patterns governing the spelling of words (Hinson, 1982). In compliance with those strategies, the findings of this study show that computer games can be a good strategy for spelling acquisition too.

As data analysis confirmed, computer games had positive influence on recall and retention of spelling. This finding supports researchers' attempts to reveal relevant relationships between writing practices learned in classrooms and gaming practices learned from playing video game. The potential relationships between writing and gaming contribute toward designing a pedagogy intended to help students understand gaming as literacy (Kuechenmeister, 2011).

As a tool for language learning/teaching, video or computer games have undoubtedly facilitated the task of spelling for learners for both inside and outside of the classroom in this study; Morgan (1979) reported that spelling games can be effective in increasing the spelling performance of students. For over 20 years researchers have been using videogames as a mean of researching individuals. According to Griffiths (2002), a professor of gambling studies in the psychology 
division of Nottingham Trent University, many of these researches also provide an insight as to why they may be useful educationally. For instance:

- Videogames can be used as research and/or measurement tools. Furthermore, as research tools they have great diversity.

- Videogames attract participation by individuals across many demographic boundaries (e.g., age, gender, ethnicity, educational status).

- Videogames can assist children in setting goals, ensuring goal rehearsal, providing feedback, reinforcement, and maintaining records of behavioral change.

- Videogames can be useful because they allow the researcher to measure performance on a very wide variety of tasks, and can be easily changed, standardized and understood.

- Videogames can be used when examining individual characteristics such as self-esteem, self-concept, goalsetting and individual differences.

- Videogames are fun and stimulating for participants. Consequently, it is easier to achieve and maintain a person's undivided attention for long periods of time. Because of the fun and excitement, they may also provide an innovative way of learning.

- Videogames can provide elements of interactivity that may stimulate learning.

- Videogames also allow participants to experience novelty, curiosity and challenge. This may stimulate learning.

- Videogames equip children with state-of-the art technology. This may help overcome technophobia (a condition well-known among many adults). Over time it may also help eliminate gender imbalance in IT use (as males tend to be more avid IT users).

- Videogames may help in the development of transferable IT skills.

- Videogames can act as simulations. These allow participants to engage in extraordinary activities and to destroy or even die without real consequences.

- Videogames may help adolescents regress to childhood play (because of the ability to suspend reality in videogame playing).

\section{Conclusion and Pedagogical Implications}

In spite of the emergence of new theories during the history of language teaching, the need for having new techniques and methods, which are context laden, is obviously cleared. To this end, in this study, we presented some games for enhancing spelling performance. As the results indicate, these games have positive influence on both retention and recall of spelling. Considering status quo games can be a good substitution for complicated tasks especially for children and young adult learners.

These kinds of activity have the potentials of adjusting different tasks with games which are exciting and surprising for children. Halliwell (1992) has summarized the benefits of meaningful games as follows: "They exploit and develop the capacity for interaction and talk, the capacity for indirect learning, the capacity for creative use of language resources, and the capacity for playing and fun" (p. 40). Accordingly, games and activities provide students with chances to examine the words closely, as well as enhancing their motivation to learn. Because of this point researchers should pay more attention to computer games in computer assisted language learning. You should bear in mind that this study was conducted just for spelling performance while computer games can adapt themselves with the teaching of different parts of the communicative competence.

The finding of this study can be useful for teachers to make the atmosphere of the class exciting for children. Also they can make student's language learning experience more meaningful as videogame can facilitate (i) discussing and sharing, (ii) following directions (understanding prepositions etc.), (iii) giving directions, (iv) answering questions, and (v) having a discussion topic with visual aides to share with others (Griffiths, 2002).

Because of the objectivity associated with computer assisted language learning, in testing of students' performance this kind of task can have a positive influence too. Consequently teachers can monitor their students meticulously.

\section{References}

Donaldson, R.P. \& Haggstrom, M.A. (2006). Changing Language Education through CALL. Routledge Studies in Computer Assisted Language Learning. London: Routledge.

Griffiths, M.D. (2002). Gambling and Gaming Addictions in Adolescence. Leicester: British Psychological Society/Blackwells.

Halliwell, S. (1992). Teaching English in the primary classroom. New York: Longman.

Hinson, A.D. (1982). Teaching Spelling for Retention. Master of Education (MEd), University of North Florida.

Keuning, J. \& Verhoeven, L. (2008). Spelling development throughout elementary grades: The Dutch case. Learning and Individual Differences, 18, 459-470.

Morgan, Q. (1979). The effect of a classroom game on spelling performance: a systematic replication. Master of Arts, Ohio State University. 
Mubireek (2003). Gender-oriented vs. gender-neutral computer games in education. Unpublished doctoral dissertation, Ohio State University.

Snowling, M.J. (1994). Towards a model of spelling acquisition: the development of some component processes. In G.D.A. Brown \& N.C. Ellis (Eds.), Handbook of spelling: Theory, process and intervention (pp. 111-128). New York: John Wiley \& Sons.

Varnhagen, C.N., McCallum, M., \& Burstow, M. (1997). Is children's spelling naturally stage-like? Reading and Writing: An Interdisciplinary Journal, 9, 451-481. doi:10.1023/A:1007903330463.

Warschauer, M. (1997). The Internet for English teaching: guidelines for teachers. TESL Reporter, 30/1, 27-33. 